\title{
Ground-glass nodules of the lung in never-smokers and smokers: clinical and genetic insights
}

\author{
Yoshihisa Kobayashi $^{1,2}$, Chiara Ambrogio ${ }^{2}$, Tetsuya Mitsudomi ${ }^{1}$ \\ ${ }^{1}$ Department of Thoracic Surgery, Kindai University Faculty of Medicine, Osaka-Sayama, Osaka 589-8511, Japan; ${ }^{2}$ Department of Medical \\ Oncology, Dana-Farber Cancer Institute, Boston, MA 02215, USA \\ Contributions: (I) Conception and design: Y Kobayashi, T Mitsudomi; (II) Administrative support: Y Kobayashi, T Mitsudomi; (III) Provision of study \\ materials or patients: Y Kobayashi, T Mitsudomi; (IV) Collection and assembly of data: All authors; (V) Data analysis and interpretation: All authors; \\ (VI) Manuscript writing: All authors; (VII) Final approval of manuscript: All authors. \\ Correspondence to: Tetsuya Mitsudomi. Department of Thoracic Surgery, Kindai University Faculty of Medicine, 377-2 Ohno-Higashi, Osaka-Sayama, \\ Osaka 589-8511, Japan. Email: mitsudom@med.kindai.ac.jp.
}

\begin{abstract}
Pulmonary ground-glass nodules (GGNs) are hazy radiological findings on computed tomography (CT). GGNs are detected more often in never-smokers. Retrospective and prospective studies have revealed that approximately $20 \%$ of pure GGNs and $40 \%$ of part-solid GGNs gradually grow or increase their solid components, whereas others remain stable for years. Most persistent or growing GGNs are lung adenocarcinomas or their preinvasive lesions. To distinguish GGNs with growth from those without growth, GGNs should be followed for at least 5 years. Lesion size and smoking history are predictors of GGN growth. Genetic analyses of resected GGNs have suggested that EGFR mutations are also predictors for growth but a subset of $K R A S$ - or $B R A F$-mutated GGNs may undergo spontaneous regression because the frequencies of $K R A S$ or $B R A F$ mutations decrease with the advance of pathological invasiveness. Although lobectomy is the standard surgical procedure for lung cancer, limited surgery such as wedge resection or segmentectomy for lung cancers $\leq 2 \mathrm{~cm}$ with consolidation/tumor ratio $\leq 0.25$ can be a viable alternative based on the recent clinical trial. Further genetic analyses and clinical trials can contribute to elucidation of the biological aspects of preinvasive adenocarcinoma and the development of less invasive management strategies for patients with GGNs.
\end{abstract}

Keywords: Adenocarcinoma; ground-glass opacity (GGO); lung cancer; subsolid nodule

Submitted Jan 01, 2018. Accepted for publication Jul 04, 2018.

doi: $10.21037 /$ tlcr.2018.07.04

View this article at: http://dx.doi.org/10.21037/tlcr.2018.07.04

\section{Introduction}

Ground-glass nodules (GGNs) on computed tomography (CT) are hazy lesions that do not obscure underlying bronchial structures or pulmonary vessels. GGNs are manifestations of both malignant and benign lesions, such as focal interstitial fibrosis, inflammation, or hemorrhage (1). However, slowly growing or stable GGNs are early lung cancers or their preinvasive lesions, atypical adenomatous hyperplasia (AAH) or adenocarcinoma in situ (AIS). AAH, AIS, and lepidic predominant lung adenocarcinomas grow along preexisting alveolar structures (2), which maintain the air space. Therefore, these lesions appear as GGNs on CT. GGNs are classified into pure GGNs and part-solid GGNs that have both ground-glass and solid components (Figure 1A).

We previously reviewed the pathological features and natural history of the GGN (3). The proportion of solid components of GGNs is closely related to pathological invasive lesions. The longest diameter of consolidation/longest diameter of tumor ratio ( $\mathrm{C} / \mathrm{T}$ ratio) is commonly used to evaluate the proportion of ground-glass components (Figure 1B). Empirically, $\mathrm{C} / \mathrm{T}$ ratio $\leq 0.5$ has been suggested as a benchmark for pathological invasiveness because the incidence of lymph node metastasis in $\leq 3 \mathrm{~cm}$ GGNs with $\mathrm{C} / \mathrm{T}$ ratio $>0.5$ ranges 

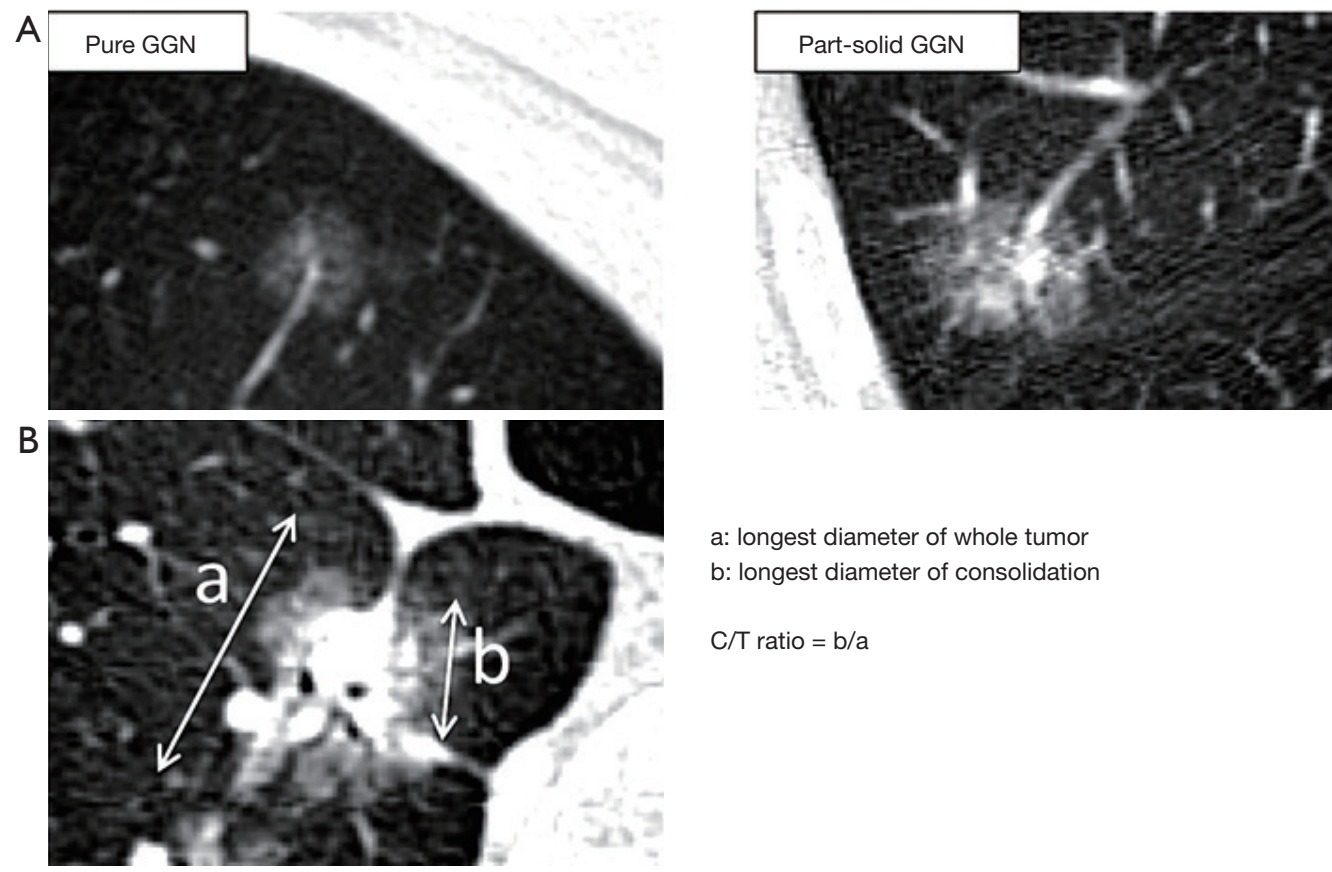

a: longest diameter of whole tumor

b: longest diameter of consolidation

$\mathrm{C} / \mathrm{T}$ ratio $=\mathrm{b} / \mathrm{a}$

Figure 1 Representative computed tomography images of pure and part-solid GGN and the definition of the consolidation/tumor (C/T) ratio. GGN, ground-glass nodule.

from $21 \%$ to $26 \%$ (4-6). Solid components of GGN on CT often contain pathologically invasive parts when analyzed under a microscope. Typically, AAH and AIS present as pure GGNs on CT, whereas minimally invasive adenocarcinoma (MIA) and lepidic invasive adenocarcinoma present as partsolid GGNs. Some GGNs exhibit gradual growth, but others remain unchanged for years.

We collected recent reports analyzing more than 100 GGNs with information on smoking history (7-15) (Table 1). In total, approximately $60 \%$ of GGNs are found in neversmokers. Although there is some inconsistency regarding the incidence of smoking status, 8 of 9 articles reported that GGNs are detected more often in never-smokers. Thus, GGN can be regarded as one of the features of lung cancer in neversmokers. In this review, we have updated recent data on GGNs in terms of smoking and genetic alterations to gain insights into the biological features of lung cancer progression and to suggest clinical management strategies for GGNs.

\section{Follow-up period of GGN}

Although GGNs often remain stable without growth for years, about $20 \%$ of pure GGNs and $40 \%$ of part-solid GGNs gradually grew or increased their solid components in our recent review summarizing four reports (3). We suggested that 3-year follow-up was a reasonable benchmark to distinguish these lesions based on the volume-doubling times of GGNs (9). In 2016, Kakinuma et al. reported more extensive results of a Japanese prospective multiinstitutional study (13). A total of 795 patients with 1,229 GGNs were assessed, and the mean follow-up period was 4.3 years. In addition to pure GGN and part-solid GGN, the authors defined heterogeneous GGN as a GGN with solid components only in the lung window but not in the mediastinal window setting. The 2 -mm growth probabilities at 5 years were $14 \%, 24 \%$, and $48 \%$ for pure, heterogeneous, and part-solid GGNs, respectively. These data are similar to the combined data in our review article (3). However, notably, some GGNs began to grow even after the 3-year follow-up. Based on these prospective data, the minimum follow-up period was extended from 3 to 5 years in the updated Fleischner Society guidelines in 2017 (16).

\section{Predictors of GGN growth}

\section{Previously reported predictors and statistical concerns}

It would be useful if we could predict which GGNs would 
Table 1 Smoking status and gender in patients with pulmonary ground-glass nodules

\begin{tabular}{|c|c|c|c|c|c|c|c|c|c|c|c|c|c|}
\hline \multirow{2}{*}{$\begin{array}{l}\text { Author } \\
\text { (reference) }\end{array}$} & \multirow{2}{*}{ Year } & \multirow{2}{*}{ GGNs } & \multirow{2}{*}{ Patients } & \multicolumn{6}{|c|}{ Smoking status } & \multicolumn{4}{|c|}{ Gender } \\
\hline & & & & Number & $\%$ & Number & $\%$ & Number & $\%$ & Number & $\%$ & Number & $\%$ \\
\hline Hiramatsu (7) & 2008 & 125 & 125 & 58 & 46 & 41 & 33 & 26 & 21 & 74 & 59 & 51 & 41 \\
\hline Chang (8) & 2013 & 122 & 89 & 30 & 34 & 59 & 66 & - & - & 16 & 18 & 73 & 82 \\
\hline Lee (10) & 2013 & 175 & 114 & 63 & 55 & 51 & 45 & - & - & 45 & 39 & 69 & 61 \\
\hline Matsuguma (11) & 2013 & $174^{*}$ & 171 & 95 & 56 & 56 & 33 & 23 & 13 & 103 & 60 & 71 & 42 \\
\hline Cho (12) & 2016 & 453 & 218 & 125 & 57 & 84 & 39 & 9 & 4 & 110 & 50 & 108 & 50 \\
\hline Kakinuma (13) & 2016 & 1229 & 795 & 474 & 60 & 317 & 40 & 4 & 0 & 454 & 57 & 341 & 43 \\
\hline Total & - & - & 1,973 & 1,153 & 58 & 748 & 38 & 75 & 4 & 1,100 & 56 & 876 & 44 \\
\hline
\end{tabular}

*, only numbers of nodules are linked to information about smoking and gender.

grow and which GGNs would remain stable without any change. Lesion diameter and past history of lung cancer were reported as predictors for the growth of GGNs $(7,8,11)$ (Figure 2). However, statistical analyses of the growth of GGNs are subject to two major concerns. First, some patients have synchronous multiple GGNs. When all GGNs are independently counted, patient characteristics, such as gender or smoking status, are overweighed because of double-counting. Second, the definition of "no-growth" as an outcome is difficult because GGNs without growth for a given period might begin to grow afterwards.

\section{Influence of smoking on GGN growth}

We conducted two independent analyses considering the above concerns (17). First, the "time to 2 -mm growth" was assessed for each lesion, and univariate and multivariate analyses using the Cox proportional hazards model were performed. To avoid possible biases in the case with multiple lesions, we performed a subsequent subanalysis dealing with only the largest lesion per patient. Next, the "incidence of 2-mm growth" was defined as an outcome, and univariate and multivariate analyses using the logistic regression model were performed. To strictly define "nogrowth", we excluded lesions that had been observed for $<3$ years based on our previous report (9). Based on the consistent results of these statistical analyses, we found that smoking, in addition to larger diameter, is a novel predictor of growth (17) (Figure 2).

Considering that GGNs are found more often in neversmokers, smoking history as a predictor of growth seems to be paradoxical. We collected recent reports describing smoking status and GGN growth $(7,8,10,12,15,17)$ (Figure 3). Combined data from six articles showed that frequency of GGN growth based on data about "growth" or "nogrowth" was significantly higher in smokers than that in never-smokers ( $26 \%$ versus $18 \%, \mathrm{P}=0.0045)$. Among the six articles, 3 reported frequencies of GGN growth at 2, 3, or 5 years based on data on "time to growth" $(11,13,17)$ (Figure 3). All data consistently showed that GGNs in smokers were more likely to grow than those in never-smokers. Notably, a prospective multi-institutional study also reported that lesion size and smoking history were factors for growth toward the appearance of solid components by multivariate analysis, although lesion size and male gender were predictors for 2-mm growth (13). Male gender and smoking were closely correlated in our previous analysis (17). Although it is not clear whether smoking cessation after diagnosis of GGN changes the clinical behavior of these GGNs, smoking cessation should be emphasized in this situation as well.

In addition to GGN growth, there is an interesting report about the influence of smoking on the new appearance of GGNs. Remy-Jardin et al. examined growth changes in 111 subjects who had undergone sequential CT 


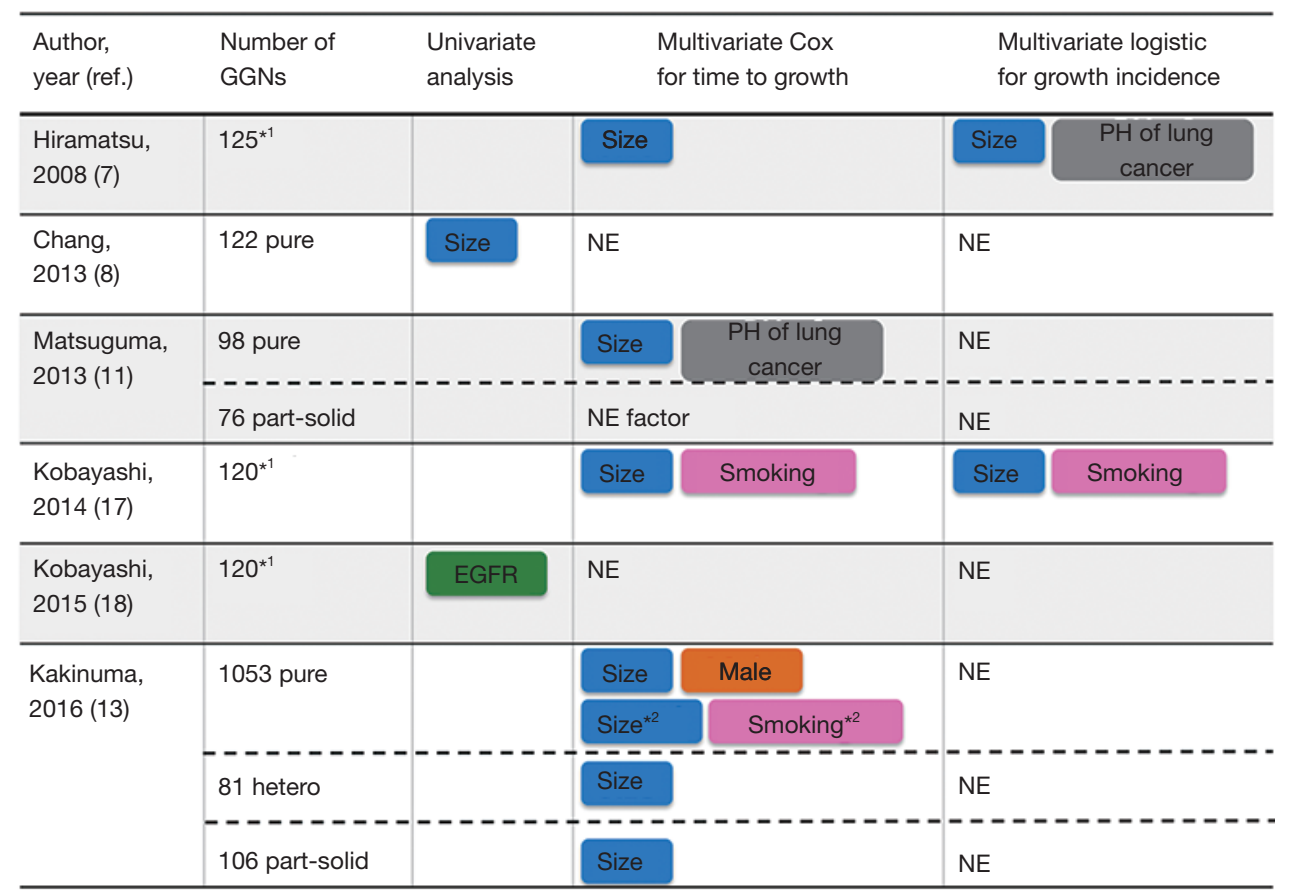

Figure 2 Summary of predictors for the growth of GGNs $(7,8,11,13,17,18)$. * 1 : pure GGNs and part-solid GGNs; *2: changes in the appearance of the solid component is defined as the outcome. NE, not evaluated; PH, past history; GGN, ground-glass nodule.

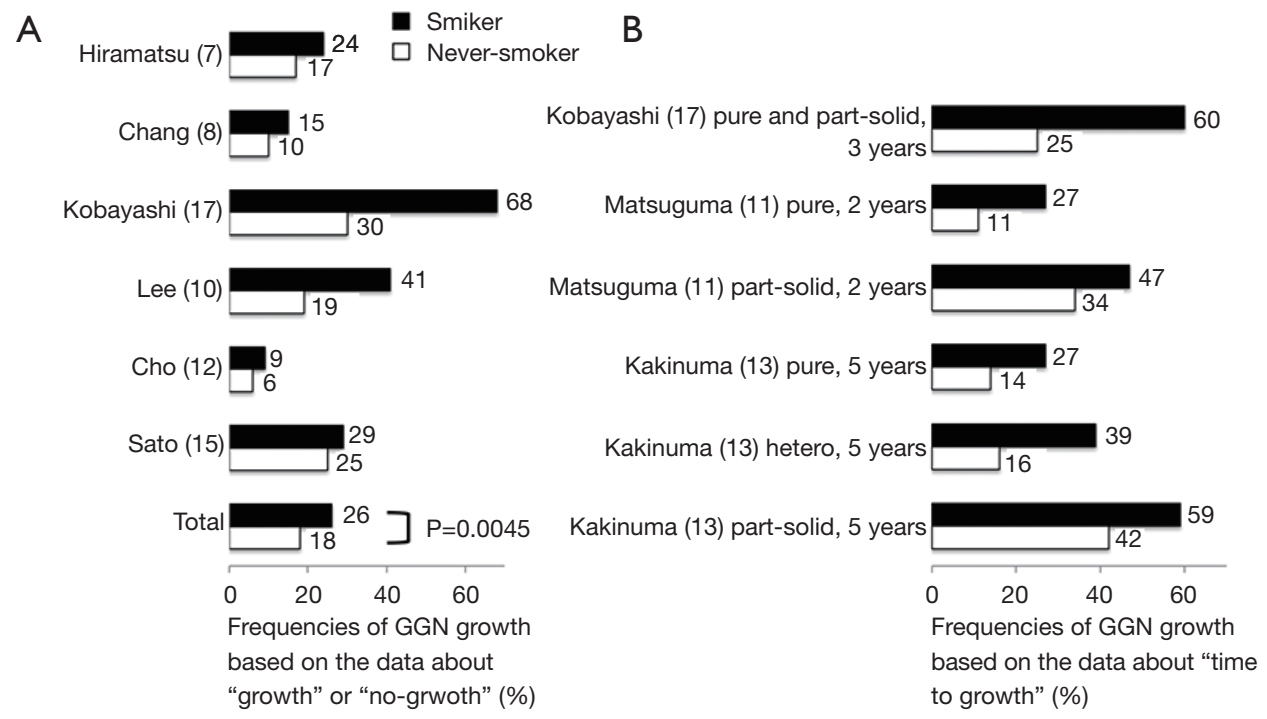

Figure 3 Frequencies of GGN growth in smokers and never-smokers $(7,8,10-13,15,17)$. Previous studies evaluated frequencies of GGN growth via two independent analyses. First, "growth" or "no-growth" was used as an outcome. Combined data from six studies were compared using Chi-square test (A). Second, "time to growth" was used as an outcome and growth rates were estimated using the KaplanMeier method. In reference 13, in addition to pure GGN and part-solid GGN, the authors defined heterogeneous GGN as a GGN with solid components only in the lung window but not in the mediastinal window setting (B). GGN, ground-glass nodule. 


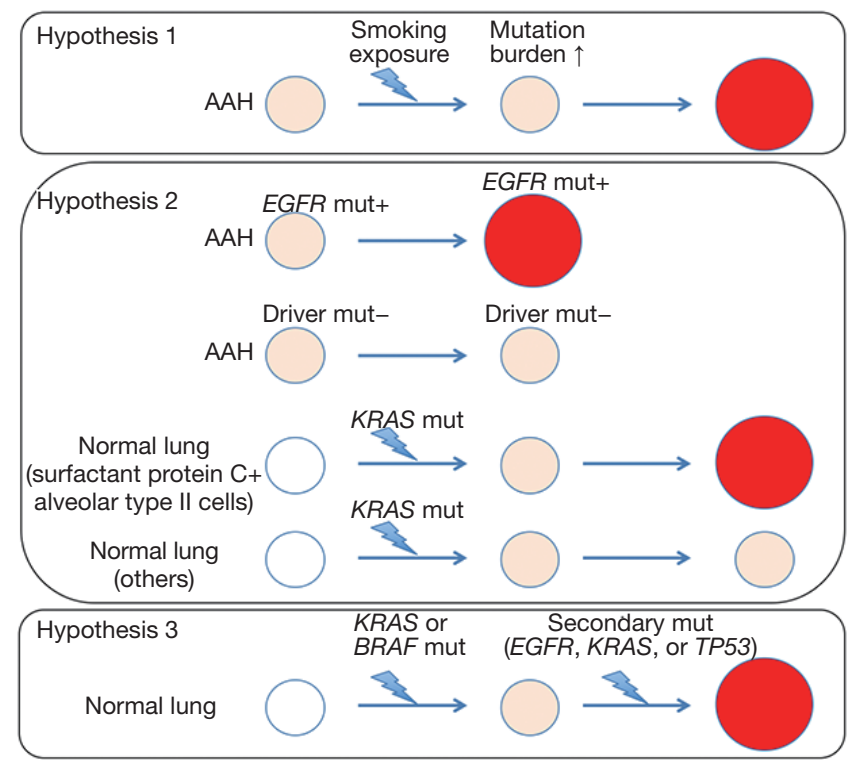

Figure 4 Hypotheses for the progression of preinvasive adenocarcinoma showing as a GGN. Hypothesis 1 suggests that smoking exposure induces a mutation burden, which contributes to the growth of the GGN. According to hypothesis 2, EGFR mutation-positive AAHs gradually grow, whereas driver mutationnegative AAHs do not progress. Among KRAS-mutant AAH, only those derived from surfactant protein $\mathrm{C}+$ alveolar type II cells may progress to invasive adenocarcinoma. Hypothesis 3 suggests that progression to invasive adenocarcinoma is induced by an initial stimulation by BRAF or KRAS mutations and the subsequent secondary mutations. GGN, ground-glass nodule; AAH, atypical adenomatous hyperplasia.

examinations over a mean period of 5.5 years. GGNs were detected in $28 \%$ of current smokers by initial evaluations. Interestingly, final evaluations detected GGNs in $42 \%$ of persistent current smokers $(\mathrm{P}=0.02)$. In contrast, the frequencies of GGNs upon initial and final evaluations were not significantly different in never-smokers (19). These data indicate that a subset of GGNs may be newly developed due to chronic exposure to smoking.

Smoking is thought to cause cancer by inducing DNA damage, which leads to somatic mutations in cancer-related genes. Alexandrov et al. collected data on somatic mutations in 5,243 cancers from the Cancer Genome Atlas (TCGA), the International Cancer Genome Consortium (ICGC), and 17 other articles to reveal the association between mutational signatures and tobacco smoking (20). In analyses of samples with information about cumulative exposure to smoking, the total numbers of base substitution mutations were positively correlated with pack-years smoked. For lung adenocarcinoma, the authors estimated that approximately 150 mutations accumulated per genome per pack-year (20). Considering these data, cumulative mutations due to smoking may induce GGN growth.

\section{The association between genetic alterations and GGN growth}

\section{EGFR mutation}

Previous studies revealed that the lesion diameter and smoking (or male gender) were predictors for the growth of GGNs as described above $(13,17)$. However, the genetic differences among GGNs with or without growth remain unclear.

Thus, we conducted genetic analyses of surgically resected GGNs (18). Genetic alterations in the EGFR, $K R A S, A L K$, and HER2 genes were evaluated in 104 GGNs, and all lesions were categorized as EGFR-, KRAS-, ALK-, or HER2-positive or quadruple-negative because of mutually exclusive relationships among driver gene mutations. The frequencies of EGFR, KRAS, ALK, and HER 2 mutations were $64 \%, 4 \%, 3 \%$, and $4 \%$, respectively. To the best of our knowledge, this study is the only study to analyze the association between genetic alterations of GGNs and their growth. Our study demonstrated that $E G F R$ mutation positivity (EGFR ${ }^{+}$) was significantly associated with growth, whereas a quadruple-negative status was associated with no-growth (Figure 4). This finding is also supported by the result that a quadruple-negative status is associated with pathological noninvasiveness.

Clinical characteristics of patients with EGFR mutation and those with GGNs seem to be similar because EGFR+ GGNs account for the majority of all GGNs. As we summarized in Table 1, GGNs are found more in nonsmokers and female. Considering that pathological features of GGNs are adenocarcinoma or its preinvasive lesions, GGNs and EGFR mutation share the same features: nonsmoker, female, and adenocarcinoma. Regarding ethical differences, it could be suspected that GGNs, especially EGFR-mutant GGNs, may be found more in East Asian than in others although there are no data.

\section{KRAS mutation}

Although the general incidence of KRAS mutations of lung adenocarcinomas in Caucasians is approximately $26 \%$ $(670 / 2,529)$, KRAS mutations tend to occur more frequently in smokers (34\%) than in never-smokers (6\%) (21). In Asians KRAS mutations were detected in only $8 \%$ 
$(429 / 5,125)$ in all cases $(22)$.

When Sakamoto et al. examined KRAS mutations in various stages of preinvasive lesions and invasive adenocarcinoma, most $K R A S$-mutated tumors were categorized into AAH: the incidences of $K R A S$ mutations were $33 \%, 12 \%$, and $8 \%$ in AAH, AIS, and MIA, respectively (23). A similar trend was observed in another study, with KRAS detection rates of $27 \%, 17 \%$, and $10 \%$ in each of the above stages of adenocarcinoma (24). Considering that the overall frequency of $K R A S$ mutations in lung adenocarcinoma is limited to $13 \%$ (25), these findings cannot be explained without assuming that some tumors and preneoplastic lesions with $K R A S$ mutations undergo spontaneous regression. This hypothesis was originally suggested by Yatabe et al. (26). One possible mechanism may be associated with the dual role of $K R A S$. Oncogenic Ras has been shown to cause senescence through the activation of the p53-p21 WAF and p16INK4ARetinoblastoma tumor-suppressor pathways $(27,28)$. Another potential explanation is that not all $K R A S$ mutant AAHs are same and the differences are derived from the cells of origin. In analyses using genetically engineered mouse models, not all $K R A S$-mutant lung cells are equally permissive to transformation: only surfactant protein $\mathrm{C}+$ alveolar type II cells are able to sustain $K R A S$-driven formation of $\mathrm{AAH}$, whereas some of which progress to adenomas and malignant adenocarcinomas regardless of surrounding microenvironment or inflammatory stimulation $(29,30)$. Additionally, the transcriptional analysis of KRAS-expressing $\mathrm{AAH}$ in vivo reveals that only a fraction retains the signature of advanced lung adenocarcinoma, whereas the others show a transcriptional profiling comparable to normal alveolar cells (31). In clinical and radiological aspects, $K R A S$-mutant GGNs on CT image seem to be very similar or identical. However, the origins of these nodules may not be identical, and this difference could cause different biological behavior: some gradually grow and others remain unchanged or disappear.

This seemingly paradoxical observation that $K R A S$ mutation is more common in AAH than in invasive adenocarcinoma is in accordance with recent reports by Sivakumar et al. and Sato et al. (32). Sivakumar et al. analyzed normal tissue, AAH, and invasive adenocarcinoma from the same patients to assess progression of lung adenocarcinoma. Undeniably, these comparisons did not directly reflect the progression of lung cancer because the discordance rate of driver mutations between invasive adenocarcinoma and paired synchronous GGNs in the same patient was $80 \%$ (24/30) (33). Yet, KRAS mutations were detected in as many as $24 \%$ (4/17) of AAHs. Sato et al. also reported a relatively high frequency of $K R A S$ mutations in Japanese patients with GGNs. KRAS mutations in codon 12 were detected in $17 \%(5 / 30)$ of resected GGNs (15). These data suggest that not all KRAS mutation-positive AAHs progress to more advanced adenocarcinomas (Figure 4).

\section{$B R A F$ mutation}

$B R A F$ mutations are detected in approximately $3 \%(18 / 687)$ of lung adenocarcinomas in Caucasians, and the V600E mutation accounts for approximately $50 \%$ of these tumors (34). BRAF mutations are categorized into 3 classes (35). Class $1 B R A F$ mutants (BRAF V600 mutations) are RAS-independent and activated as monomers. Class 2 mutants are RAS-independent and activated as dimers. Class 3 mutants are RAS-dependent and have impaired kinase activity, in other words, they are kinase-dead. Similar to the low frequency of KRAS mutations in Asians, $B R A F$ mutations were detected in only $0.5 \%$ (26/5,125) of Asians (22).

In the above-mentioned study by Sivakumar et al., the frequencies of $E G F R, K R A S$, and $B R A F$ mutations in invasive adenocarcinoma were $47 \%(8 / 17), 6 \%(1 / 17)$, and $0 \%$, respectively. However, the $\mathrm{BRAF}^{+}$rate in AAHs was as high as $29 \%(5 / 17)$ (32). Four of the five AAHs exhibited $B R A F$ K601E mutation, and the other AAH harbored $B R A F$ N581S mutation. These mutations belong to class 2 and class 3 , respectively. These non-V600E mutations have been previously noted in lung cancer $(36,37)$ and demonstrated to be oncogenic drivers (38). Sivakumar et al. hypothesized that progression to invasive adenocarcinoma is induced by an initial stimulation by the $B R A F$ or $K R A S$ mutations and the subsequent secondary mutations (32). Although this hypothesis is interesting, there must be an advantage to lose the mutated allele during progression in these cases. In other words, $K R A S$ or $B R A F$ are not acting as driver oncogenes. Confirmation of this hypothesis through analyses of sequential biopsied samples is essential.

Similar to AAHs with $K R A S$ mutations, not all $B R A F$ mutated AAHs develop into invasive adenocarcinomas. The similar roles of $K R A S$ and $B R A F$ in the mitogen-activated protein kinase (MAPK) pathway might be associated with this phenomenon.

\section{Timing of surgery for GGN}

The criteria of surgery for GGNs vary depending on the guideline. According to the guidelines of the American College of Chest Physicians, surgical resection is 

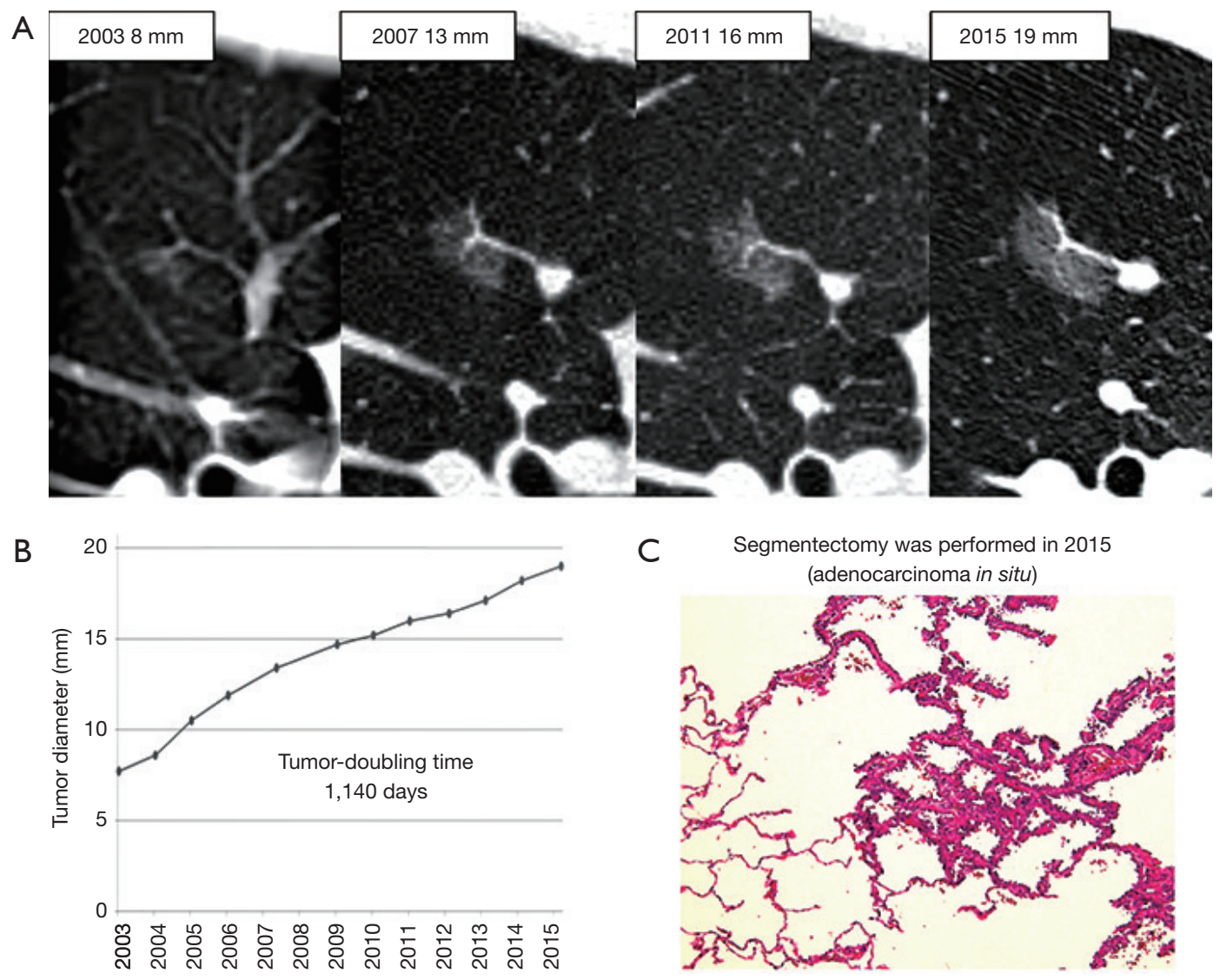

Figure 5 Computed tomography images and pathological features of representative pure GGN. (A) Pure GGN was detected in a 49-yearold male with previous smoking history of 4 pack years; This GGN slowly increased in diameter without appearance of solid components; (B) tumor-doubling time was 1,140 days; (C) segmentectomy was performed in 2015 after 12-years of follow-up. This tumor was diagnosed as adenocarcinoma in situ (hematoxylin-eosin staining, $\times 40$ ). GGN, ground-glass nodule.

recommended for GGNs that meet any of the following conditions: any GGN with growth or the development of solid components, pure GGNs $>10 \mathrm{~mm}$ with confirmed persistence, part-solid GGNs $>8 \mathrm{~mm}$ with confirmed persistence, or part-solid GGNs $>15 \mathrm{~mm}$ without followup (39). The Fleischner Society recommends that pure GGNs with the development of solid components or grow and persistent part-solid nodules with solid components $\geq 6$ $\mathrm{mm}$ should be resected (16).

However, it is doubtful whether immediate surgery is necessary for all GGNs showing growth. In our case with pure GGN, segmentectomy was performed after 12 years' follow-up. Although the GGN had continued to grow for 12 years, tumor-doubling time was as long as 1,140 days and pathological diagnosis revealed that it was still AIS without invasive lesions (Figure 5). It is unclear when this type of tumor begins to invade surrounding structures and threaten patient's life and whether surgery is really necessary. Therefore, the balance between the benefits of surgery and life expectancy should be considered particularly in elderly patients.

\section{Extent of surgery for GGN}

\section{A Clinical trial on radiological criteria}

A prospective multi-institutional study was conducted by the Japan Clinical Oncology Group (JCOG) to identify radiological criteria that predict the pathologic noninvasiveness of clinical IA lung cancer arising in the periphery of the lung (JCOG 0201) (40). The consolidation/ tumor ratio $(\mathrm{C} / \mathrm{T}$ ratio) was used to evaluate the proportion of the ground-glass component. This study revealed that the specificities for the diagnosis of pathological noninvasiveness were $96.4 \%$ and $98.7 \%$ for lesions $\leq 3 \mathrm{~cm}$ with 

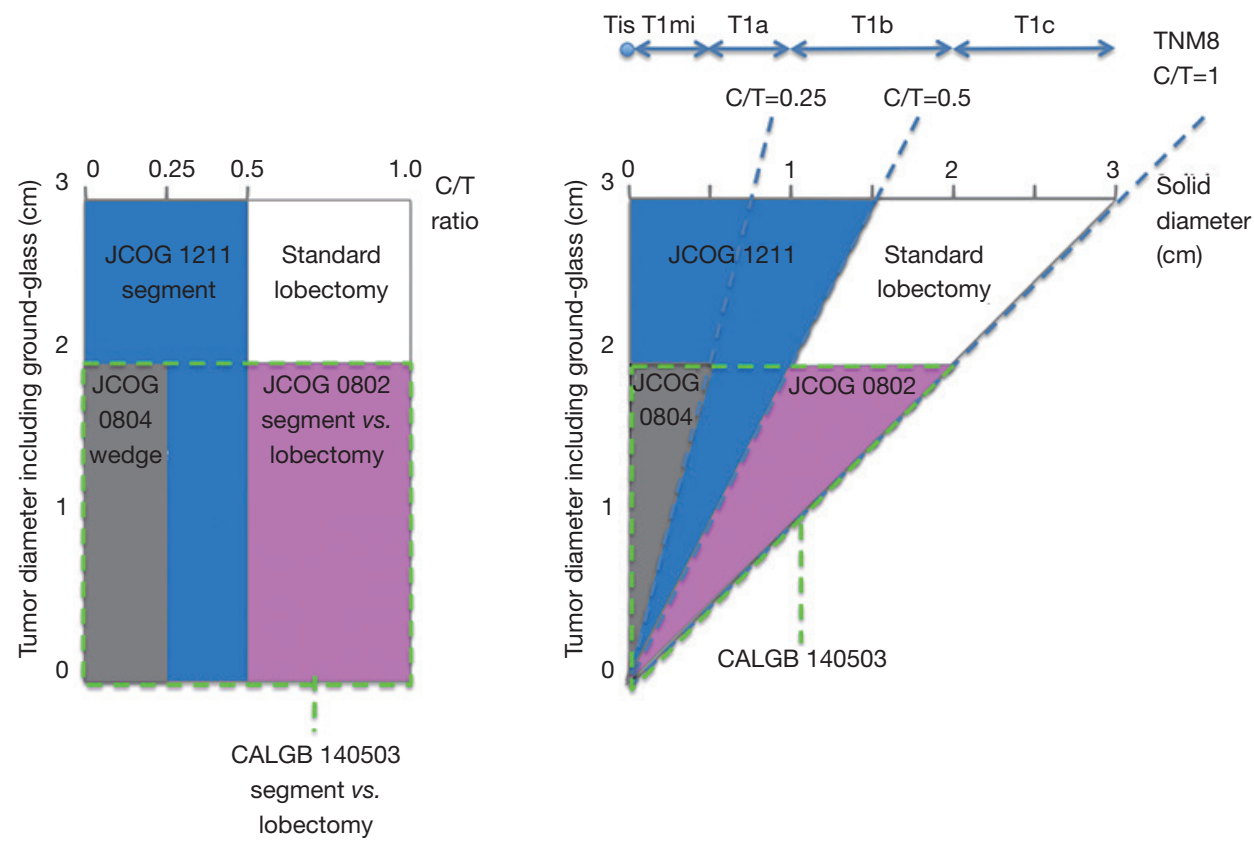

Figure 6 Summary of Japanese clinical trials evaluating the efficacy of limited surgery for GGNs. The inclusion criteria of each trial are summarized on the left side. The 8th TNM classifications using the solid diameter were combined with the abovementioned inclusion criteria using the consolidation/tumor ratio (C/T ratio). GGN, ground-glass nodule.

a $\mathrm{C} / \mathrm{T}$ ratio $\leq 0.5$ ( $>50 \%$ ground-glass component) and lesions $\leq 2 \mathrm{~cm}$ with a $\mathrm{C} / \mathrm{T}$ ratio $\leq 0.25$ ( $>75 \%$ ground-glass component), respectively (40).

The long-term survival rate of patients in the JCOG 0201 trial who underwent lobectomy and lymph node dissection was reported. The overall and relapse-free 5 -year survival rates of all the patients were $90.6 \%$ and $84.7 \%$, respectively. When a lesion size $\leq 3 \mathrm{~cm}$ with a $\mathrm{C} / \mathrm{T}$ ratio $\leq 0.5$ was used as a cutoff, the 5 -year overall survival rates of radiological noninvasive and invasive adenocarcinomas were $96.7 \%$ and $88.9 \%$, respectively $(\mathrm{P}<0.001)$. With the use of lesions $\leq 2$ $\mathrm{cm}$ with $\mathrm{C} / \mathrm{T}$ ratios $\leq 0.25$, the 5 -year overall survival rates of radiological noninvasive and invasive adenocarcinomas were $97.1 \%$ and $92.4 \%$, respectively $(\mathrm{P}=0.259)(41)$.

\section{Clinical trials on limited surgery for GGNs}

Although the standard treatment for operable non-small cell lung cancer is lobectomy with dissection of ipsilateral hilar and mediastinal lymph nodes (42), retrospective data support the efficacy and less invasive nature of limited surgery such as segmentectomy or wedge resection for GGNs. Based on the results of the JCOG 0201 study, three clinical trials evaluating the efficacy of limited surgery were conducted (Figure 6). JCOG 0802 is a phase III trial comparing lobectomy and segmentectomy for lung cancer $\leq 2 \mathrm{~cm}$ with C/T ratio $>0.5$ (43), which is similar to the Cancer and Leukemia Group B (CALGB) 140503 trial in the United States (44). JCOG 0804 is a phase III nonrandomized confirmatory study of wedge resection for lung cancer $\leq 2 \mathrm{~cm}$ with a $\mathrm{C} / \mathrm{T}$ ratio $\leq 0.25$ (45). JCOG 1211 is a confirmatory Phase III trial of segmentectomy for lung cancer $\leq 3 \mathrm{~cm}$ with a $\mathrm{C} / \mathrm{T}$ ratio $\leq 0.5$ (46).

The results of JCOG 0804 were presented in ASCO 2017 (47). The surgical procedure is basically set as wedge resection, but segmentectomy is allowed when the surgical margin is insufficient $(<5 \mathrm{~mm})$ or when the tumor is histologically invasive. The 5 -year recurrence-free survival was $99.7 \%$, which met the primary endpoint, and no local relapse was noted.

The TNM classification was revised in 2017. Regarding the measurement of a GGN, the diameter of the solid component within the part-solid nodule on CT instead of the whole tumor size should be measured for staging (48). The application of the results of the JCOG trials to the 8th TNM classification is somewhat complicated because the JCOG trials measure the C/T ratio while the TNM classification addresses the direct measurement of the solid 
component (Figure 6).

\section{Conclusions}

Recent clinical and genetic data mark the beginning of elucidating biological aspects of GGNs. GGNs are often found in never-smokers, but smoking is a predictor of growth. In terms of genetic alterations, EGFR mutation, which is associated with never-smokers, is also a predictor of growth, whereas a subset of GGNs with smoke-related KRAS or BRAF mutations may undergo spontaneous regression. Additionally, driver mutation-negative GGNs tend to remain unchanged. Although these data superficially appear paradoxical, further genetic analyses and clinical trials could contribute to deeper understanding of preinvasive adenocarcinoma and the development of less invasive management strategies for GGNs.

\section{Acknowledgements}

The authors thank Dr. Shigeaki Moriura (Checkup Center, Daiyukai Daiichi Hospital) for providing CT images.

Funding: This work was supported by a Grant-in-Aid for Scientific Research from the Japan Society for the Promotion of Science (16K19989 to Y. Kobayashi).

\section{Footnote}

Conflicts of Interest: The authors have no conflicts of interest to declare.

\section{References}

1. Park CM, Goo JM, Lee HJ, et al. Nodular groundglass opacity at thin-section CT: histologic correlation and evaluation of change at follow-up. Radiographics 2007;27:391-408.

2. Travis WD, Brambilla E, Noguchi M, et al. International association for the study of lung cancer/american thoracic society/european respiratory society international multidisciplinary classification of lung adenocarcinoma. J Thorac Oncol 2011;6:244-85.

3. Kobayashi Y, Mitsudomi T. Management of ground-glass opacities: should all pulmonary lesions with ground-glass opacity be surgically resected? Transl Lung Cancer Res 2013;2:354-63.

4. Aoki T, Tomoda Y, Watanabe H, et al. Peripheral lung adenocarcinoma: correlation of thin-section CT findings with histologic prognostic factors and survival. Radiology 2001;220:803-9.

5. Matsuguma H, Yokoi K, Anraku M, et al. Proportion of ground-glass opacity on high-resolution computed tomography in clinical T1 N0 M0 adenocarcinoma of the lung: A predictor of lymph node metastasis. J Thorac Cardiovasc Surg 2002;124:278-84.

6. Nakata M, Sawada S, Yamashita M, et al. Objective radiologic analysis of ground-glass opacity aimed at curative limited resection for small peripheral non-small cell lung cancer. J Thorac Cardiovasc Surg 2005;129:122631.

7. Hiramatsu M, Inagaki T, Inagaki T, et al. Pulmonary ground-glass opacity (GGO) lesions-large size and a history of lung cancer are risk factors for growth. J Thorac Oncol 2008;3:1245-50.

8. Chang B, Hwang JH, Choi YH, et al. Natural history of pure ground-glass opacity lung nodules detected by lowdose CT scan. Chest 2013;143:172-8.

9. Kobayashi Y, Fukui T, Ito S, et al. How long should small lung lesions of ground-glass opacity be followed? J Thorac Oncol 2013;8:309-14.

10. Lee SW, Leem CS, Kim TJ, et al. The long-term course of ground-glass opacities detected on thin-section computed tomography. Respir Med 2013;107:904-10.

11. Matsuguma H, Mori K, Nakahara R, et al. Characteristics of subsolid pulmonary nodules showing growth during follow-up with CT scanning. Chest 2013;143:436-43.

12. Cho J, Kim ES, Kim SJ, et al. Long-Term Follow-up of Small Pulmonary Ground-Glass Nodules Stable for 3 Years: Implications of the Proper Follow-up Period and Risk Factors for Subsequent Growth. J Thorac Oncol 2016;11:1453-9.

13. Kakinuma R, Noguchi M, Ashizawa K, et al. Natural History of Pulmonary Subsolid Nodules: A Prospective Multicenter Study. J Thorac Oncol 2016;11:1012-28.

14. Lee JH, Park CM, Lee SM, et al. Persistent pulmonary subsolid nodules with solid portions of $5 \mathrm{~mm}$ or smaller: Their natural course and predictors of interval growth. Eur Radiol 2016;26:1529-37.

15. Sato Y, Fujimoto D, Morimoto T, et al. Natural history and clinical characteristics of multiple pulmonary nodules with ground glass opacity. Respirology 2017;22:1615-21.

16. MacMahon H, Naidich DP, Goo JM, et al. Guidelines for Management of Incidental Pulmonary Nodules Detected on CT Images: From the Fleischner Society 2017. Radiology 2017;284:228-43.

17. Kobayashi Y, Sakao Y, Deshpande GA, et al. The 
association between baseline clinical-radiological characteristics and growth of pulmonary nodules with ground-glass opacity. Lung Cancer 2014;83:61-6.

18. Kobayashi Y, Mitsudomi T, Sakao Y, et al. Genetic features of pulmonary adenocarcinoma presenting with groundglass nodules: the differences between nodules with and without growth. Ann Oncol 2015;26:156-61.

19. Remy-Jardin M, Edme JL, Boulenguez C, et al. Longitudinal follow-up study of smoker's lung with thinsection CT in correlation with pulmonary function tests. Radiology 2002;222:261-70.

20. Alexandrov LB, Ju YS, Haase K, et al. Mutational signatures associated with tobacco smoking in human cancer. Science 2016;354:618-22.

21. Dogan S, Shen R, Ang DC, et al. Molecular epidemiology of EGFR and KRAS mutations in 3,026 lung adenocarcinomas: higher susceptibility of women to smoking-related KRAS-mutant cancers. Clin Cancer Res 2012;18:6169-77.

22. Li S, Li L, Zhu Y, et al. Coexistence of EGFR with KRAS, or BRAF, or PIK3CA somatic mutations in lung cancer: a comprehensive mutation profiling from 5125 Chinese cohorts. Br J Cancer 2014;110:2812-20.

23. Sakamoto H, Shimizu J, Horio Y, et al. Disproportionate representation of KRAS gene mutation in atypical adenomatous hyperplasia, but even distribution of EGFR gene mutation from preinvasive to invasive adenocarcinomas. J Pathol 2007;212:287-94.

24. Yoshida Y, Shibata T, Kokubu A, et al. Mutations of the epidermal growth factor receptor gene in atypical adenomatous hyperplasia and bronchioloalveolar carcinoma of the lung. Lung Cancer 2005;50:1-8.

25. Kosaka T, Yatabe Y, Endoh H, et al. Mutations of the epidermal growth factor receptor gene in lung cancer: biological and clinical implications. Cancer Res 2004;64:8919-23.

26. Yatabe Y, Borczuk AC, Powell CA. Do all lung adenocarcinomas follow a stepwise progression? Lung Cancer 2011;74:7-11.

27. Collado M, Gil J, Efeyan A, et al. Tumour biology: senescence in premalignant tumours. Nature 2005;436:642.

28. Karnoub AE, Weinberg RA. Ras oncogenes: split personalities. Nat Rev Mol Cell Biol 2008;9:517-31.

29. Mainardi S, Mijimolle N, Francoz S, et al. Identification of cancer initiating cells in K-Ras driven lung adenocarcinoma. Proc Natl Acad Sci U S A 2014;111:255-60.

30. Sutherland KD, Song JY, Kwon MC, et al. Multiple cells-of-origin of mutant K-Ras-induced mouse lung adenocarcinoma. Proc Natl Acad Sci U S A 2014;111:4952-7.

31. Ambrogio C, Gomez-Lopez G, Falcone M, et al. Combined inhibition of DDR1 and Notch signaling is a therapeutic strategy for KRAS-driven lung adenocarcinoma. Nat Med 2016;22:270-7.

32. Sivakumar S, Lucas FAS, McDowell TL, et al. Genomic Landscape of Atypical Adenomatous Hyperplasia Reveals Divergent Modes to Lung Adenocarcinoma. Cancer Res 2017;77:6119-30.

33. Wu C, Zhao C, Yang Y, et al. High Discrepancy of Driver Mutations in Patients with NSCLC and Synchronous Multiple Lung Ground-Glass Nodules. J Thorac Oncol 2015;10:778-83.

34. Paik PK, Arcila ME, Fara M, et al. Clinical characteristics of patients with lung adenocarcinomas harboring BRAF mutations. J Clin Oncol 2011;29:2046-51.

35. Yao Z, Yaeger R, Rodrik-Outmezguine VS, et al. Tumours with class 3 BRAF mutants are sensitive to the inhibition of activated RAS. Nature 2017;548:234-8.

36. Cancer Genome Atlas Research Network. Comprehensive molecular profiling of lung adenocarcinoma. Nature 2014;511:543-50. Erratum in: Nature 2014;514:262. Rogers, K [corrected to Rodgers, K]. Author Correction: Comprehensive molecular profiling of lung adenocarcinoma. [Nature. 2018]

37. Marchetti A, Felicioni L, Malatesta S, et al. Clinical features and outcome of patients with non-small-cell lung cancer harboring BRAF mutations. J Clin Oncol 2011;29:3574-9.

38. Nieto P, Ambrogio C, Esteban-Burgos L, et al. A Braf kinase-inactive mutant induces lung adenocarcinoma. Nature 2017;548:239-43.

39. Gould MK, Donington J, Lynch WR, et al. Evaluation of individuals with pulmonary nodules: when is it lung cancer? Diagnosis and management of lung cancer, 3rd ed: American College of Chest Physicians evidence-based clinical practice guidelines. Chest 2013;143:e93S-120S.

40. Suzuki K, Koike T, Asakawa T, et al. A prospective radiological study of thin-section computed tomography to predict pathological noninvasiveness in peripheral clinical IA lung cancer (Japan Clinical Oncology Group 0201). J Thorac Oncol 2011;6:751-6.

41. Asamura H, Hishida T, Suzuki K, et al. Radiographically determined noninvasive adenocarcinoma of the lung: survival outcomes of Japan Clinical Oncology Group 0201. J Thorac Cardiovasc Surg 2013;146:24-30. 
42. Ginsberg RJ, Rubinstein LV. Randomized trial of lobectomy versus limited resection for T1 N0 non-small cell lung cancer. Lung Cancer Study Group. Ann Thorac Surg 1995;60:615-22; discussion 622-3.

43. A phase III randomised trial of lobectomy versus limited resection (segmentectomy) for small ( $2 \mathrm{~cm}$ or less) peripheral non-small cell lung cancer (JCOG0802/ WJOG4607L) [University hospital medical information network web site]. Accessed January 7, 2018. Available online: https://upload.umin.ac.jp/cgi-open-bin/ctr_e/ctr_ view.cgi? recptno=R000002300

44. A Phase III Randomized Trial of Lobectomy Versus Sublobar Resection for Small $(\leq 2 \mathrm{~cm})$ Peripheral NonSmall Cell Lung Cancer. Accessed January 17, 2018. Available online: https://clinicaltrials.gov/ct2/show/ NCT00499330

45. A phase III non-randomized confirmatory study of Limited Surgical Resection for Peripheral Early Lung Cancer Defined with Thoracic Thin-section Computed Tomography (JCOG0804/WJOG4507L). Accessed January 17, 2018. Available online: https://

Cite this article as: Kobayashi Y, Ambrogio C, Mitsudomi T. Ground-glass nodules of the lung in never-smokers and smokers: clinical and genetic insights. Transl Lung Cancer Res 2018;7(4):487-497. doi: 10.21037/tlcr.2018.07.04 upload.umin.ac.jp/cgi-open-bin/ctr_e/ctr_view. cgi? recptno=R000002262

46. A phase III Confirmatory Trial of Segmentectomy for Clinical T1N0 Lung Cancer Dominant with Ground Glass Opacity based on Thin-section Computed Tomography (JCOG1211). Accessed January 17, 2018. Available online: https://upload.umin.ac.jp/cgi-open-bin/ctr_e/ctr_view. cgi?recptno=R000013286

47. Suzuki K, Watanabe S, Wakabayashi M, et al. A nonrandomized confirmatory phase III study of sublobar surgical resection for peripheral ground glass opacity dominant lung cancer defined with thoracic thin-section computed tomography (JCOG0804/WJOG4507L). J Clin Oncol 2017:35: abstr 8561.

48. Travis WD, Asamura H, Bankier AA, et al. The IASLC Lung Cancer Staging Project: Proposals for Coding T Categories for Subsolid Nodules and Assessment of Tumor Size in Part-Solid Tumors in the Forthcoming Eighth Edition of the TNM Classification of Lung Cancer. J Thorac Oncol 2016;11:1204-23. 